\title{
Power Allocation for Joint Interweave and Underlay Cognitive Radio Systems with Arbitrary Input Distributions
}

\author{
Ahmed Sohail, Mohammed Al-Imari, Pei Xiao, Barry G. Evans \\ Centre for Communication Systems Research, University of Surrey, Guildford, GU2 7XH, UK \\ Email: \{a.sohail,m.al-imari,p.xiao,b.evans\}@ surrey.ac.uk
}

\begin{abstract}
In the literature, the Gaussian input is assumed in power optimization algorithms. However, this assumption is unrealistic, whereas practical systems use Finite Symbol Alphabet (FSA) input, (e.g., M-QAM). In this paper, we consider the optimal power for joint interweave and underlay CR systems given FSA inputs. We formulated our problem as convex optimization and solved it through general convex optimization tools. We observed that the total SU transmit power is always less than the power budget and remains in interference limited region only over the considered distance range. Therefore, we rederive optimal power with interference constraint only in order to reduce the complexity of the algorithm by solving it analytically. Numerical results reveal that, for the considered distance range, the transmit power saving and the rate gain with the proposed algorithm is in the range $16-92 \%$ and $7-34 \%$, respectively, depending on the modulation scheme (i.e., BPSK, QPSK and 16-QAM) used.
\end{abstract}

Index Terms-Cognitive Radio, OFDM, Finite Symbol Alphabet, MMSE, Mutual Information.

\section{INTRODUCTION}

The static frequency band allocations is one of the main reasons for spectrum under-utilization and, therefore, draws attention towards schemes that implement dynamic spectrum allocation. It has been shown by Ofcom survey [1] that different areas of the UK experience spectrum under-utilization for significant period of time. Likewise, study published by the FCC [2] also indicates under-utilization of spectrum by $13.1 \%$ and $35 \%$ in New York City and downtown Washington DC respectively, below $3 \mathrm{GHz}$. These findings clearly suggest the need for flexible spectrum sharing schemes in order to cope with physical spectrum shortage. One way to address this problem is to allow Secondary User (SU) to opportunistically access the licensed spectrum under a Cognitive Radio (CR) framework, given an acceptable interference to the Primary User (PU) [3]. The underlay, overlay and interweave spectrum sharing (ISS) schemes for the CR systems have been discussed in details in [4], [5]. In the ISS scheme, the spectrum is only accessible by the SU once it is left by the PU. However, when $\mathrm{PU}$ is present, spectrum can still be accessed via underlay scenario. Therefore, in this paper, the focus of our study is the joint ISS and USS scheme due to its ability to achieve higher data rates compared to ISS and USS schemes only.

The Orthogonal Frequency Division Multiplexing (OFDM) modulation scheme which is used to mitigate multi-path propagation problems [6] in current wireless communication standards and services, is also well suited for CR systems. It offers the ability to monitor the PU spectral activity and flexibility to allocate unused spectrum dynamically among SU subcarriers [7].

One of the limitations of the CR system is the mutual interference caused in between the PU and the SU. It impacts the performance of both the PU and the SU. This can be dealt with dynamic power allocation schemes in OFDM based CR systems that aims to reduce the mutual interference by adjusting the transmit power on each subcarrier of the SU. However, traditional power allocation schemes (i.e., waterfilling etc.) do not consider interference constraint leading to unacceptable interference to the PU. Therefore, a judicious power allocation scheme is required which takes into consideration the interference introduced by the SU's subcarriers into the PU band in addition to the channel condition. To maximize the SU data rate under given constraints, different power allocation schemes for ISS [8], [9] and USS [10] schemes have been proposed in the literature, assuming Gaussian input distributions. Although, the Gaussian input distribution is theoretically optimal for mutual information maximization, however: (i) it is not valid assumption for practical system; (ii) the power optimized for Gaussian input distribution is sub-optimal when it is used for for Finite Symbol Alphabet (FSA) transmission and (iii) the capacity achieved based on the Gaussian input is unbounded in the Signal-to-Noise Ratio (SNR). On the other hand, a sub-optimal FSA input distribution is more realistic assumption for practical systems and is bounded in the SNR, i.e., $\log _{2}(\mathcal{F})$, where $\mathcal{F}$ denotes the FSA set. As achievable rates attained by the FSA input are always lower than the capacity attained by the Gaussian input, achievable rates attained by the FSA input are approximated by the capacity attained by the Gaussian input, using a SNR gap model as proposed in [11]. However, the approximation is not valid at high SNRs due to the large gap and its inability to predict the rate saturation point. One possible solution to address this limitation is to derive the optimal power with FSA input distribution, as given in [12]. However, in this work, authors considered a non-cognitive scenario, whereas in interference limited CR systems, the same power allocation algorithm cannot be applied due to mutual interference, which degrades the performance of both the PU and the SU. To address this problem, optimal power in OFDM based CR 
systems given an FSA input distribution is derived in [13], [14].

The aforementioned work addresses power allocation algorithms in ISS scheme. In [15], optimal power is evaluated for joint ISS and USS scheme but again with the Gaussian input assumption. To the best of our knowledge, no work has been done to derive and evaluate optimal power with arbitrary input distributions in joint scheme. The contributions of this paper are summarized as follows;

- We formulate a convex optimization problem by considering interference and power budget constraints and accordingly derive the optimal power allocation for an FSA input distribution by capitalizing on the relationship between mutual information and Minimum Mean Square Error (MMSE) [16]. Although, the problem is solvable through general optimization algorithms but the complexity of the algorithm is high for practical applications. Moreover, from simulation results we found that the total transmit power of the $\mathrm{SU}$ is below the maximum transmit power and remains in interference limited region over the considered distance range. This gives us relaxation to rederive the optimal power with interference constraint only in order to solve the problem analytically which reduces the complexity of the algorithm.

- We show that there is a wastage of transmit power if the conventionally optimized power with the Gaussian input assumption is used for the FSA transmission. Whereas the optimal power allocation derived by the proposed scheme leads to a significant power saving. Moreover, we show that the Gaussian optimized power results in a reduced transmission rate compared to the proposed scheme. It is because as Gaussian optimized power becomes higher than the proposed optimal power, it consequently nullify more subcarriers in comparison to the proposed optimal power and thus more subcarriers are wasted.

The organization of the remainder paper is as follows. In Sec. II we present system model and optimal power allocation policy for OFDM based CR systems is proposed in Sec. III. Simulation results of the proposed scheme are illustrated in Sec. IV. and finally, conclusions are drawn in Sec. V.

\section{SySTEM MODEL}

In our system model, we assume a single-cell wireless system in the downlink path. We further assume that the PU and the SU transceivers coexist in the same geographical location as shown in Fig. 1. A joint ISS and USS scheme is illustrated in Fig. 2, where all subcarriers whether occupied or unoccupied by the PU are considered for power allocation by the SU that employs OFDM modulation scheme for transmission. The total bandwidth $B$ is divided into $L$ subcarriers containing $M$ occupied subcarriers and $N$ unoccupied subcarriers, each subcarrier having bandwidth $\Delta f \mathrm{~Hz}$. The channel gain between the SU transmitter and the SU receiver, i.e., $g_{\ell}$ is assumed to be known a-priori at the SU transmitter via a pilot assisted channel estimation.

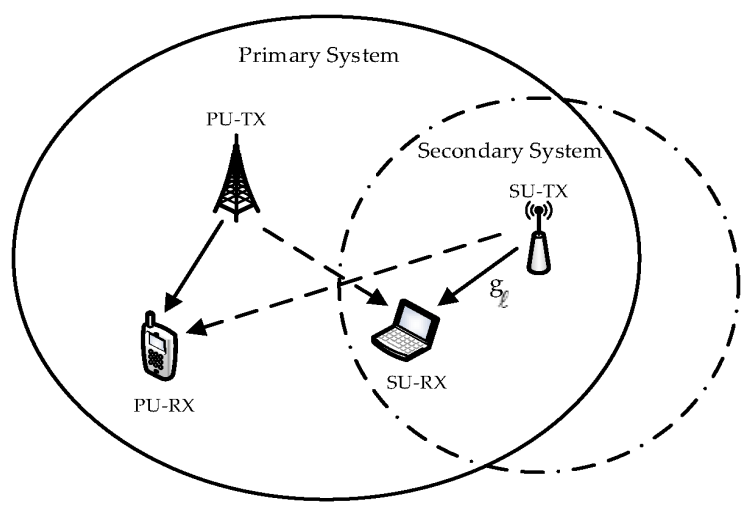

Fig. 1. A single cell cognitive radio System Model

In the joint scheme, the transmit power of the $\mathrm{SU}$ is based on the interference threshold and the total power constraints imposed by the PU. Therefore, to maximize the SU achievable data rate under given constraints, we derive an optimal power with FSA input distributions based on the convex optimization problem. The key to solve the optimum power allocation problem is the relationship between mutual information and MMSE and is given by

$$
\frac{d I\left(s n r_{\ell}, S\right)}{d\left(\operatorname{snr}_{\ell}\right)}=m m s e\left(\operatorname{snr}_{\ell}, S\right)
$$

in Eq. (1), $I($.$) represents mutual information and s n r_{\ell}=$ $p_{\ell} g_{\ell}$. Whereas $p_{\ell}$ is the optimal power for $\ell$ th subcarrier and $S$ denotes an arbitrary input distribution, (e.g., FSA or Gaussian). We remove $S$ from equations in the rest of the paper, whenever no ambiguity arises. There are two types of interference in $\mathrm{CR}$ system (i) interference from the $\mathrm{SU}$ into the $\mathrm{PU}$ and (ii) interference from the PU into the SU. Since, our objective is to protect the PU from an unacceptable interference, we will only consider interference introduced by the SU into the PU band [8] and will treat interference introduced by the PU into the SU as noise. The interference introduced by the SU to the $\mathrm{PU}$ is given by

$J_{\ell, m}\left(d_{\ell, m}, p_{\ell}\right)=p_{\ell} \Phi_{\ell, m}=p_{\ell} T_{s} \int_{\left(d_{\ell, m}-\frac{1}{2}\right) \Delta f}^{\left(d_{\ell, m}+\frac{1}{2}\right) \Delta f}\left(\frac{\sin \pi f T_{s}}{\pi f T_{s}}\right)^{2} d f$,

where

$$
\Phi_{\ell, m}=\frac{\partial J_{\ell, m}}{\partial p_{\ell}}=T_{s} \int_{\left(d_{\ell, m}-\frac{1}{2}\right) \Delta f}^{\left(d_{\ell, m}+\frac{1}{2}\right) \Delta f}\left(\frac{\sin \pi f T_{s}}{\pi f T_{s}}\right)^{2} d f
$$

and $J_{\ell, m}$ is the interference introduced by the $\ell$ th subcarrier of the SU into the $m$ th PU band for the joint ISS and USS scheme, $T_{s}$ is the symbol duration, $\Delta f$ is the frequency spacing between two adjacent subcarriers, $d_{\ell, m}$ represents the spectral distance between the $\ell$ th subcarrier of the SU and the $m$ th PU band. 


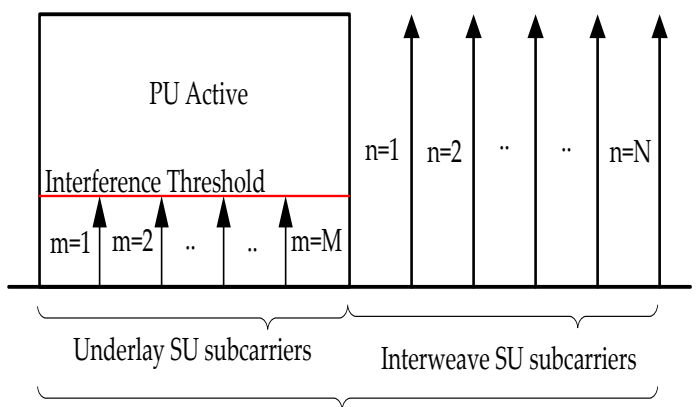

$\mathrm{L}=\mathrm{M}+\mathrm{N}=$ Total number of SU subcarriers

Fig. 2. Graphical representation of joint ISS and USS scheme

\section{Optimal Power Allocation Policy}

The objective is to calculate an optimal power in the joint ISS and USS scheme with an arbitrary input distribution that maximizes the mutual information of the $\mathrm{SU}$, provided that the total power budget and interference introduced into the PUs' band does not exceed a certain level. This problem can be defined as an optimization problem as follows

$$
[P 1] \quad \max _{p_{\ell}} \sum_{\ell=1}^{L} I\left(p_{\ell} g_{\ell}\right),
$$

subject to

$$
\begin{gathered}
\sum_{\ell=1}^{L} p_{\ell} \Phi_{\ell, m} \leq \frac{\tau_{t h}^{m}}{\Omega} \quad m=1,2, \cdots, M, \\
\sum_{\ell=1}^{L} p_{\ell} \leq P_{\max }, \\
p_{\ell} \geq 0 \quad \ell=1,2, \cdots, L,
\end{gathered}
$$

where $P_{\max }$ and $\tau_{t h}^{m}$ represent the maximum SU transmit power and interference threshold on the $m$ th subcarrier prescribed by the PU, respectively. Whereas, $\Omega$ is the path loss and is a function of the distance between the SU transmitter and the PU receiver.

Theorem 1: The optimal power with an arbitrary input distribution that maximizes SU data rate is as follows

$$
p_{\ell}^{\star}= \begin{cases}\frac{1}{g_{\ell}} \mathrm{mmse}^{-1}\left(\frac{\chi_{\ell}+\mu}{g_{\ell}}\right) & \text { if } g_{\ell}>\chi_{\ell}+\mu, \\ 0 & \text { if } g_{\ell} \leq \chi_{\ell}+\mu,\end{cases}
$$

where

$$
\chi_{\ell}=\sum_{m=1}^{M} \lambda_{m} \Phi_{\ell, m}
$$

and $\lambda_{m}, \mu$ are the Lagrange multipliers for interference Eq. (4) and power budget Eq. (5) constraints, respectively.
Proof: The optimization problem [P1] is convex [17] because (i) the summation of the mutual information, the mutual information is concave [18, section 2.7], preserves the concavity, therefore, the objective function Eq. (3) is concave and (ii) the constraints Eq. (4), Eq. (5) and Eq. (6) are linear functions of the power. With any positive power, $p_{\ell}>0$, the Slater condition is satisfied that satisfies the interference and power budget constraints. Therefore, the KKT conditions are necessary and sufficient for the optimal solution. The Lagrangian for the primal problem is as follows;

$$
\begin{aligned}
\mathcal{L}(\mathbf{p}, \boldsymbol{\lambda}, \mu, \boldsymbol{\nu})= & -\sum_{\ell=1}^{L} I\left(p_{\ell} g_{\ell}\right)+\mu\left(\sum_{\ell=1}^{L} p_{\ell}-P_{\max }\right) \\
& +\sum_{m=1}^{M} \lambda_{m}\left(\sum_{\ell=1}^{L} p_{\ell} \Phi_{\ell, m}-\frac{\tau_{t h}^{m}}{\Omega}\right)-\nu_{\ell} p_{\ell} .
\end{aligned}
$$

To find the global solution, we solve the KKT conditions as follows.

Gradient of Lagrangian with respect to $p_{\ell}^{\star}$ vanishes:

$$
\begin{gathered}
-\frac{\partial I\left(p_{\ell}^{\star} g_{\ell}\right)}{\partial p_{\ell}^{\star}}+\sum_{m=1}^{M} \lambda_{m} \frac{\partial\left(p_{\ell}^{\star} \Phi_{\ell, m}\right)}{\partial p_{\ell}^{\star}}+\mu-\nu_{\ell}=0, \\
\nu_{\ell} \geq 0, \quad p_{\ell}^{\star} \geq 0, \quad \lambda_{m} \geq 0, \quad \mu \geq 0, \\
\nu_{\ell} p_{\ell}^{\star}=0, \\
\lambda_{m}\left(\sum_{\ell=1}^{L} p_{\ell} \Phi_{\ell, m}\right)=0, \quad \mu\left(\sum_{\ell=1}^{L} p_{\ell}\right)=0 .
\end{gathered}
$$

Using the fact that $\frac{\partial I\left(p_{\ell}^{\star} g_{\ell}\right)}{\partial p_{\ell}^{\star}}=g_{\ell} \operatorname{mmse}\left(p_{\ell}^{\star} g_{\ell}\right)$, Eq. (9) can be rewritten as

$$
-g_{\ell} \operatorname{mmse}\left(p_{\ell}^{\star} g_{\ell}\right)+\chi_{\ell}+\mu-\nu_{\ell}=0 .
$$

From (10) and (13), we have

$$
g_{\ell} \operatorname{mmse}\left(p_{\ell}^{\star} g_{\ell}\right) \leq \chi_{\ell}+\mu,
$$

and from (11) and (13), we obtain

$$
p_{\ell}^{\star}\left\{\chi_{\ell}+\mu-g_{\ell} \operatorname{mmse}\left(p_{\ell}^{\star} g_{\ell}\right)\right\}=0 .
$$

Consequently, if $p_{\ell}^{\star}>0$ then from Eq. (15) we have $\chi_{\ell}+\mu=g_{\ell} \operatorname{mmse}\left(p_{\ell}^{\star} g_{\ell}\right)$, therefore

$$
p_{\ell}^{\star}=\frac{1}{g_{\ell}} \mathrm{mmse}^{-1}\left(\frac{\chi_{\ell}+\mu}{g_{\ell}}\right) .
$$

Since mmse $\left(p_{\ell}^{\star} g_{\ell}\right)<1$ when $p_{\ell}^{\star}>0$, we obtain from Eq. (14) $g_{\ell}>\chi_{\ell}+\mu$. On the other hand, as $\operatorname{mmse}(0)=1$, if $p_{\ell}^{\star}=0$, we have from Eq. (14) $g_{\ell} \leq \chi_{\ell}+\mu$.

The optimal power in [P1] can not be solved analytically, therefore, we used CVX, a package for specifying and solving convex programs [19], [20]. Simulation results are presented in the Sec. IV, where Fig. 3 (line styles) shows the total SU trasmit power by considering interference and power budget constraints [Eqs. (4), (5)]. It can be observed from this figure 
that the SU transmit power is always less than the given $P_{\max }$, i.e., total SU transmit power remains in interference limited region over the considered distance range.

\section{A. Optimal Power Allocation Policy for Interference Limited Region}

Although the optimal power problem in [P1] is solvable via general convex optimization algorithm tools, convergence to the algorithm is slow for practical applications. In order to reduce the complexity of the algorithm and to solve the optimal power analytically, we re-derive the optimal power by only considering the interference constraint. The problem can be re-defined as an optimization problem as follows

$$
[P 2] \quad \max _{p_{\ell}} \sum_{\ell=1}^{L} I\left(p_{\ell} g_{\ell}\right),
$$

subject to

$$
\begin{gathered}
\sum_{\ell=1}^{L} p_{\ell} \Phi_{\ell, m}=\frac{\tau_{t h}^{m}}{\Omega} \quad m=1,2, \cdots, M, \\
p_{\ell} \geq 0 \quad \ell=1,2, \cdots, L,
\end{gathered}
$$

Theorem 2: The optimal power with an arbitrary input distribution that maximizes SU data rate is as follows

$$
p_{\ell}^{\star}= \begin{cases}\frac{1}{g_{\ell}} \mathrm{mmse}^{-1}\left(\frac{\chi_{\ell}}{g_{\ell}}\right) & \text { if } g_{\ell}>\chi_{\ell}, \\ 0 & \text { if } g_{\ell} \leq \chi_{\ell},\end{cases}
$$

where $\lambda_{m}$ are the Lagrange multipliers for interference constraints and can be solved using numerical methods, (i.e., Newton, bisection or secant) by solving the following equation

$$
\sum_{\ell=1}^{\left(L, g_{\ell}>\chi_{\ell}\right)} \frac{1}{g_{\ell}} \mathrm{mmse}^{-1}\left(\frac{\chi_{\ell}}{g_{\ell}}\right) \Phi_{\ell, m}-\frac{\tau_{t h}^{m}}{\Omega}=0 .
$$

Proof: As the optimization problem is convex and the Slater condition is satisfied, the KKT conditions are necessary and sufficient for the optimal solution. The Lagrangian for the primal problem is as follows

$$
\begin{aligned}
\mathcal{L}(\mathbf{p}, \boldsymbol{\lambda}, \boldsymbol{\nu})= & -\sum_{\ell=1}^{L} I\left(p_{\ell} g_{\ell}\right)-\nu_{\ell} p_{\ell} \\
& +\sum_{m=1}^{M} \lambda_{m}\left(\sum_{\ell=1}^{L} p_{\ell} \Phi_{\ell, m}-\frac{\tau_{t h}^{m}}{\Omega}\right) .
\end{aligned}
$$

We solve the KKT conditions as follows

Gradient of the Lagrangian with respect to $p_{\ell}^{\star}$ vanishes:

$$
\begin{gathered}
-\frac{\partial I\left(p_{\ell}^{\star} g_{\ell}\right)}{\partial p_{\ell}^{\star}}+\sum_{m=1}^{M} \lambda_{m} \frac{\partial\left(p_{\ell}^{\star} \Phi_{\ell, m}\right)}{\partial p_{\ell}^{\star}}-\nu_{\ell}=0, \\
\nu_{\ell} \geq 0, \quad p_{\ell}^{\star} \geq 0, \quad \lambda_{m} \geq 0 \\
\nu_{\ell} p_{\ell}^{\star}=0
\end{gathered}
$$

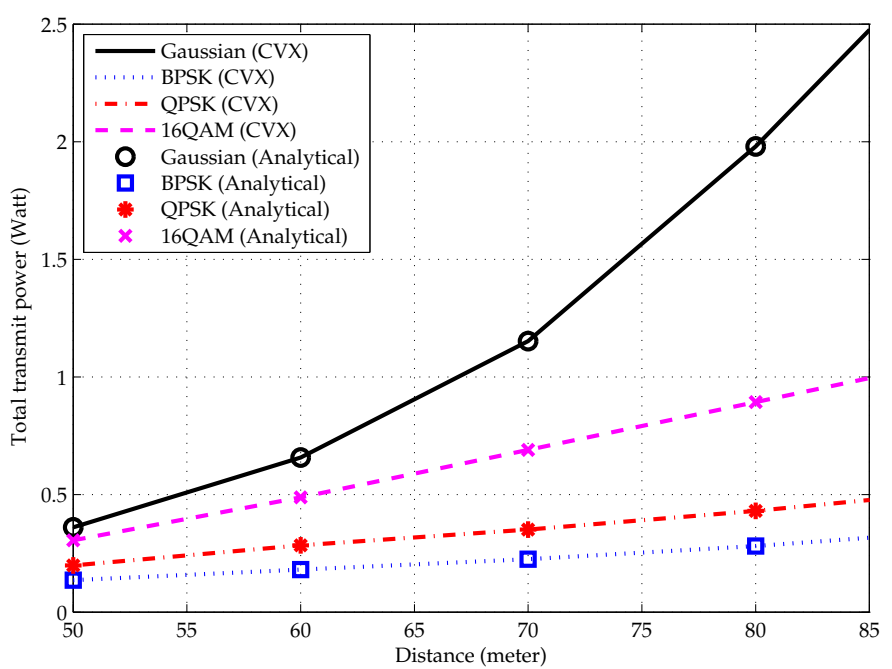

Fig. 3. Comparison between total SU transmit power with Gaussian and FSA inputs vs distance

By following the same steps as in Theorem 1 to solve the KKT conditions, we have

$$
p_{\ell}^{\star}= \begin{cases}\frac{1}{g_{\ell}} \mathrm{mmse}^{-1}\left(\frac{\chi_{\ell}}{g_{\ell}}\right) & \text { if } g_{\ell}>\chi_{\ell}, \\ 0 & \text { if } g_{\ell} \leq \chi_{\ell},\end{cases}
$$

The advantage of Theorem 2 is that the optimal power can be calculated analytically and complexity is reduced compared to the optimal power in Theorem 1. Moreover, it is worth mentioning that our optimal power derivation is generic and is valid for any input distributions, whereas, in [15] the optimal power is derived only for the Gaussian input distribution.

\section{Evaluation OF OFDM BASEd CR SYSTEM}

In this section, we show the optimal power and achievable data rate for the Gaussian and the FSA input distribution using Monte Carlo Simulations, for OFDM based CR network via joint ISS and USS scheme as depicted in Fig. 2. In our simulations, we have adopted LTE parameters for the SU transmission and assume that the total of $20 \mathrm{MHz}$ bandwidth is divided into 100 resource blocks (RBs) containing 50 occupied (underlay) RBs and 50 unoccupied (interweave) RBs [21]. A simplified path loss model has been considered, i.e., $Q\left(\frac{r_{0}}{r}\right)$ [22], where $Q$ is constant, $r_{0}$ (reference distance) and $r$ (distance between the SU transmitter and the PU receiver) is defined in meters. The values of $T_{s}, P_{\max }$ and $r_{0}$ are $4 \mu \mathrm{s}, 5$ Watts (maximum power of Micro-cell) and 50 meters, respectively, and $\tau_{t h}$ is assumed to be equivalent to thermal noise per RB. The $r$ ranges from 50 to 85 meters and accordingly $\tau_{t h}$ value increases. Additionally, an IEEE 802.11 multipath channel model with root mean square delay spread of $50 \mathrm{~ns}$ has been assumed. The results are averaged over 2000 


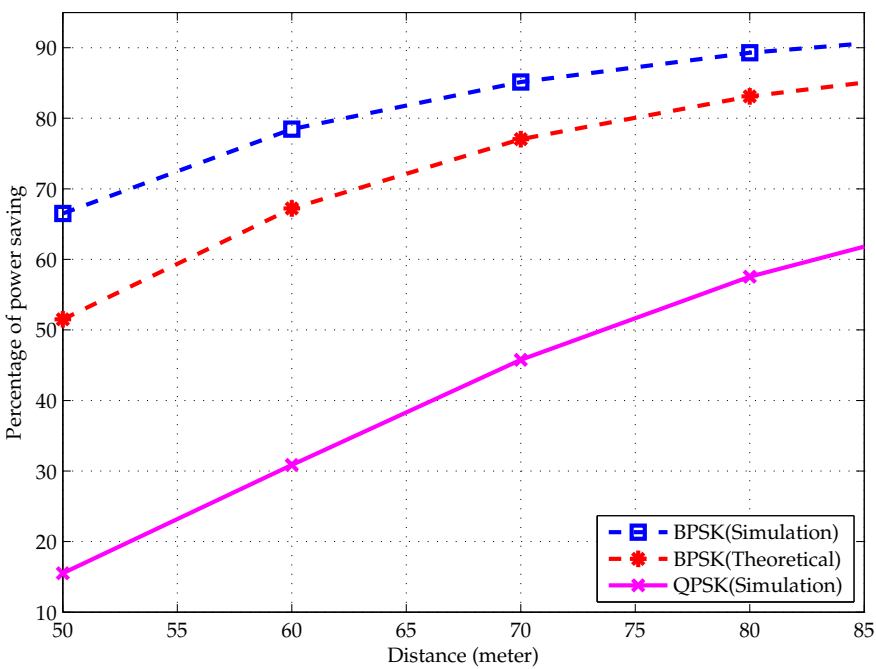

Fig. 4. Percentage of power saving for different modulation schemes vs distance

snapshots. We denote the total transmit $\operatorname{power}\left(P^{\star}=\sum_{\ell=1}^{L} p_{\ell}^{\star}\right)$ with Gaussian inputs as $P_{G}^{\star}$ and with FSA inputs as $P_{F}^{\star}$.

In Fig. 3, we calculate the optimal power in [P1] via CVX, a package for specifying and solving convex programs [19], [20] and in [P2] via analytically. We observe from this figure that (i) analytical results (marker types) coincide exactly with the CVX results (line styles), which shows accuracy of our algorithm; (ii) $P_{G}^{\star}$ is always greater than $P_{F}^{\star}$ over the considered distance range. It has been noted that power difference gap increases in proportion to the distance metric values meaning that, the power difference gap is smaller at lower distance values compared to higher ones. This could be explained as: (i) the increase in $P_{F}^{\star}$ is marginal at higher distance values because MI reaches an upper bound limit; (ii) on the other hand, $P_{G}^{\star}$ increases with increasing distance because MI under $P_{G}^{\star}$ has no upper bound limit. Moreover, it has been observed that at a fix distance metric value, we see that $P_{F}^{\star}$ increases with increasing modulation scheme, (i.e., from BPSK to 16-QAM). The optimal power allocation is dependent and specific for every modulation scheme. It would result in power inefficiency if one tries to transmit BPSK signal with the power which is optimized for 16-QAM. Therefore, for efficient power utilization, power must be optimized according to the actual employed modulation scheme.

In Fig. 4, a Gaussian optimized power has been compared against our proposed power allocation scheme in terms of power saving (i.e., $\frac{P_{G}^{\star}-P_{F}^{\star}}{P_{G}^{\star}} 100 \%$ for BPSK, QPSK and 16QAM versus distance). It can be clearly seen that a significant power saving has been achieved by our proposed optimal power $P_{F}^{\star}$ in comparison to $P_{G}^{\star}$. The transmit power saving for distance values ranging from $50 \mathrm{~m}$ to $85 \mathrm{~m}$ has found out to be $66.5-92 \%, 51.5-90 \%$ and $15.6-61 \%$ for BPSK, QPSK and 16-QAM inputs, respectively.

Fig. 5 shows a comparison of achieved data rate for the FSA transmission between the Gaussian optimized power and the power optimized based on the actual modulation scheme. It

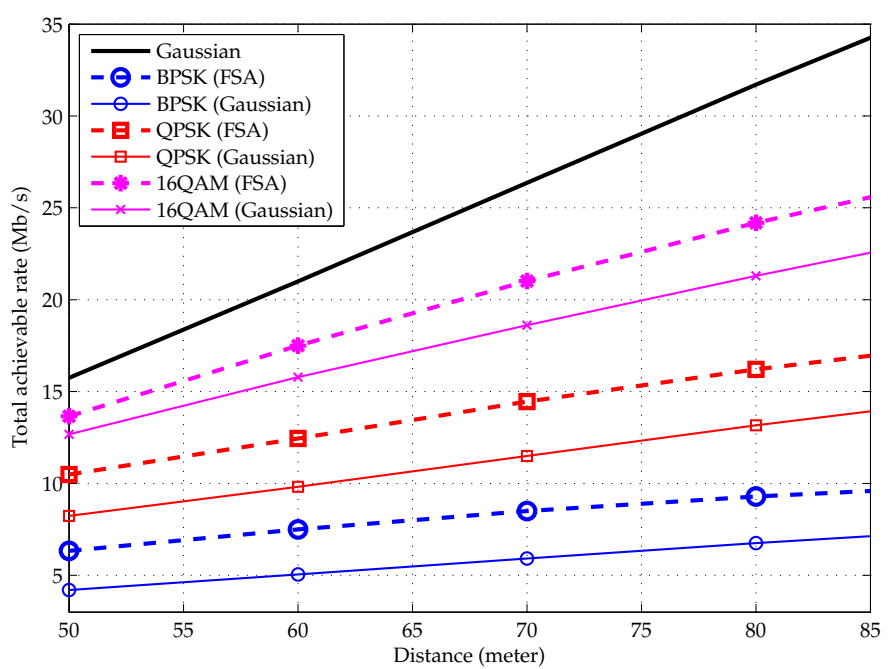

Fig. 5. Comparison between achievable data rate under Gaussian and FSA inputs vs distance

can be seen that the proposed optimal power allocation scheme achieves higher data rates compared to the traditional Gaussian power allocation scheme. In Fig. 6, we show the impact of distance metric ranging from $50 \mathrm{~m}$ to $85 \mathrm{~m}$ on percentage of rate gain for the CR system. The BPSK, QPSK and 16QAM inputs achieve a rate gain of $34.2-27.5 \%, 25.7-21.7 \%$ and $6.6-12.2 \%$, respectively. The higher interference caused by the co-existence of PU and SU in the CR system limits the percentage rate gain for the Gaussian optimized power. It is because as $P_{G}^{\star}$ becomes higher than the $P_{F}^{\star}$, it consequently nullify more subcarriers in comparison to $P_{F}^{\star}$ and thus more subcarriers are wasted. This allows the FSA input to achieve a better performance in terms of overall rate gain.

\section{CONCLUSION}

The optimal power allocation for the joint interweave and underlay spectrum sharing scheme with arbitrary input distributions has been proposed in this paper. By capitalizing the relationship between mutual information and MMSE using standard convex optimization techniques, an optimal power has been derived that maximizes the SU data rate with given constraints. Based on the numerical results, it can be seen that the SU total power allocation is less than the power budget for the considered distance range. In order to reduce the complexity of the algorithm and to solve the optimal power analytically, we re-derived the optimal power without considering the power budget constraint. It has been shown that analytical results and general optimization algorithm results coincide with each other which shows accuracy of our proposed algorithm. Moreover, it is worth highlighting that: (i) our optimal power with finite symbol alphabet input significantly outperforms the optimal power with Gaussian inputs in terms of transmit power saving and achievable data rate; (ii) with fix distance metric, the optimal transmit power with the finite symbol alphabet input increases as the modulation order increases. Based on the aforementioned findings 


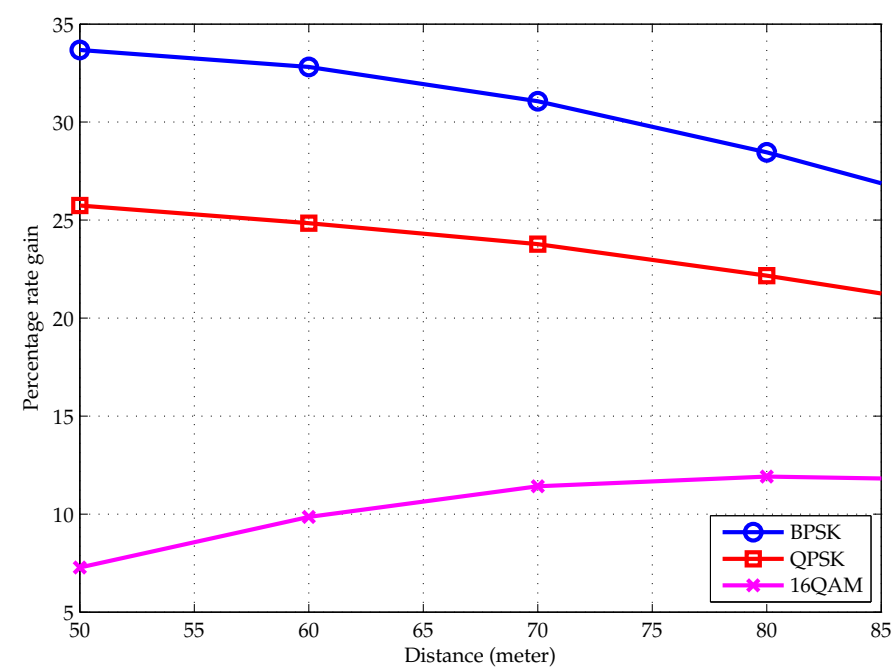

Fig. 6. Percentage of rate gain for different modulation schemes vs distance

we concluded that, by using the proposed power allocation scheme, spectrum and energy efficiency can both be improved. Secondly, to achieve a desired energy efficiency, the power should be optimized according to the employed modulation scheme.

\section{ACKNOWLEDGMENT}

This work has been supported by the India UK Advance Technology Center of Excellence in Next Generation Networks, Systems and Services (www.iu-atc.com).

\section{REFERENCES}

[1] A. Shukla, "Cognitive radio technology-a study for Ofcom," Tech.Rep. 830000143, QinetiQ Ltd, Hampshire, UK, 2006.

[2] "Federal communications commission: Spectrum policy task force report," http://fjallfoss.fcc.gov, nov. 2002.

[3] A. Sendonaris, E. Erkip, and B. Aazhang, "User cooperation diversity. part I. system description," IEEE Trans. Commun., vol. 51, no. 11, pp. 1927-1938, Nov. 2003.

[4] S. Srinivasa and S. Jafar, "Cognitive radios for dynamic spectrum access - the throughput potential of cognitive radio: A theoretical perspective," IEEE Commun. Mag., vol. 45, no. 5, pp. 73-79, May 2007.

[5] A. Goldsmith, S. Jafar, I. Maric, and S. Srinivasa, "Breaking spectrum gridlock with cognitive radios: An information theoretic perspective," vol. 97, no. 5, pp. 894-914, 2009.

[6] H. Mahmoud, T. Yucek, and H. Arslan, "OFDM for cognitive radio: merits and challenges," IEEE Wireless Commun., vol. 16, no. 2, pp. 6-15, April 2009.

[7] T. Weiss, J. Hillenbrand, A. Krohn, and F. Jondral, "Mutual interference in OFDM-based spectrum pooling systems," 59th IEEE Vehicular Technology Conference, vol. 4, pp. 1873 - 1877, May 2004.

[8] G. Bansal, M. Hossain, and V. Bhargava, "Optimal and suboptimal power allocation schemes for OFDM-based cognitive radio systems," IEEE Trans. Wireless Commun., vol. 7, no. 11, pp. 4710-4718, Nov. 2008.

[9] Z. Hasan, G. Bansal, E. Hossain, and V. Bhargava, "Energy-efficient power allocation in OFDM-based cognitive radio systems: A risk-return model," IEEE Trans. Wireless Commun., vol. 8, no. 12, pp. 6078-6088, Dec. 2009.

[10] P. Wang, M. Zhao, L. Xiao, S. Zhou, and J. Wang, "Power allocation in OFDM-based cognitive radio systems," IEEE Global Communications Conference, pp. 4061-4065, 2007.

[11] B. Devillers, J. Louveaux, and L. Vandendorpe, "Bit and power allocation for goodput optimization in coded parallel subchannels with ARQ," IEEE Trans. Signal Process., vol. 56, no. 8, pp. 3652-3661, 2008.
[12] A. Lozano, A. Tulino, and S. Verdu, "Optimum power allocation for parallel Gaussian channels with arbitrary input distributions," IEEE Trans. Inf. Theory, vol. 52, no. 7, pp. 3033-3051, July 2006.

[13] A. Sohail, M. Al-Imari, P. Xiao, and B. Evans, "Optimal power allocation for OFDM based cognitive radio systems with arbitrary input distributions," Accepted in IEEE Vehicular Technology Conference, 2013.

[14] — , "Optimal power allocation for MIMO-OFDM based cognitive radio systems with arbitrary input distributions," Accepted in IEEE Personal Indoor and Mobile Radio Communications, 2013.

[15] G. Bansal, O. Duval, and F. Gagnon, "Joint overlay and underlay power allocation scheme for OFDM-based cognitive radio systems," IEEE Vehicular Technology Conference, pp. 1-5, 2010.

[16] D. Guo, S. Shamai, and S. Verdu, "Mutual information and minimum mean-square error in gaussian channels," IEEE Trans. Inf. Theory, vol. 51, no. 4, pp. 1261-1282, April 2005.

[17] Z.-Q. Luo and W. Yu, "An introduction to convex optimization for communications and signal processing," IEEE J. Sel. Areas Commun., vol. 24, no. 8, pp. 1426-1438, Aug. 2006.

[18] T. M. Cover and J. A. Thomas, Elements of Information Theor. Hoboken, New Jersey: Wiley, 2006.

[19] M. Grant and S. Boyd, "CVX: Matlab software for disciplined convex programming, version 2.0 beta," http://cvxr.com/cvx, Sep. 2013.

[20] - "Graph implementations for nonsmooth convex programs," in Recent Advances in Learning and Control, ser. Lecture Notes in Control and Information Sciences, V. Blondel, S. Boyd, and H. Kimura, Eds. Springer-Verlag Limited, 2008, pp. 95-110.

[21] J. Zyren and W. McCoy, "Overview of the 3GPP long term evolution physical layer," Freescale Semiconductor, Inc., white paper, July 2007.

[22] A. Goldsmith, "Wireless communications." New York: Cambridge University Press, 2005. 